TRANSACTIONS OF THE

AMERICAN MATHEMATICAL SOCIETY

Volume 354, Number 10, Pages 4245-4260

S 0002-9947(02)03045-3

Article electronically published on May 23, 2002

\title{
CONTACT REDUCTION
}

\author{
CHRISTOPHER WILLETT
}

\begin{abstract}
In this article I propose a new method for reducing co-oriented contact manifold $M$ equipped with an action of a Lie group $G$ by contact transformations. With a certain regularity and integrality assumption the contact quotient $M_{\mu}$ at $\mu \in \mathfrak{g}^{*}$ is a naturally co-oriented contact orbifold which is independent of the contact form used to represent the given contact structure. Removing the regularity and integrality assumptions and replacing them with one concerning the existence of a slice, which is satisfied for compact symmetry groups, results in a contact stratified space; i.e., a stratified space equipped with a line bundle which, when restricted to each stratum, defines a co-oriented contact structure. This extends the previous work of the author and E. Lerman.
\end{abstract}

\section{INTRODUCTION}

In this article I propose a new method for reducing a co-oriented contact manifold $M$ under the action of a Lie group $G$ by contact transformations. With a certain regularity and integrality assumption (see Lemma 3.6 and Theorem 1) the contact quotient $M_{\mu}$ at $\mu \in \mathfrak{g}^{*}$ is a naturally co-oriented contact orbifold which is independent of the contact form used to represent the given contact structure. Removing the regularity and integrality assumption and replacing it with one concerning the existence of a slice, which is satisfied for compact symmetry groups, results in a contact stratified space; i.e., a stratified space equipped with a line bundle which, when restricted to each stratum, defines a co-oriented contact structure. This extends the previous work of the author and E. Lerman ([LW]).

The earliest notion of a contact quotient that I am aware of is in the work of V. Guillemin and S. Sternberg, where it appears in the guise of co-isotropic reduction of symplectic cones (GS1]). The Guillemin-Sternberg quotient is based on the reduction of foliations and is akin to the Kazhdan-Kostant-Sternberg quotient in symplectic geometry. The new contact quotient is akin to the MarsdenWeinstein-Meyer quotient. The two models differ, but are related: the GuilleminSternberg quotient fibers over a co-adjoint orbit with typical fiber the new quotient. The Guillemin-Sternberg quotient is described in a later section. Independently of Guillemin-Sternberg, C. Albert developed a model for contact reduction which is valid at all $\mu \in \mathfrak{g}^{*}$ but depends upon the contact form used to represent the given contact structure $([\mathrm{Al}])$.

Received by the editors November 21, 2001.

2000 Mathematics Subject Classification. Primary 53D10, 53D20.

The author was supported by a National Science Foundation graduate Vertical Integration of Research and Education fellowship and the American Institute of Mathematics.

(C)2002 American Mathematical Society 
Acknowledgements. The author would like to thank Eugene Lerman for suggesting this line of investigation and innumerable discussions on the material while at the American Institute of Mathematics during the fall of 2000 and for reading several preliminary versions of the paper. The author would also like to thank Susan Tolman for making several helpful suggestions concerning the presentation of the material.

A note on notation. The notation in this paper will be consistent with that used in [LW].

\section{Group aCtions on CONTACT MANifolds}

Before proceeding to the proof of the main theorems of the paper, we first recall the relevant and essential facts about contact manifolds and group actions on contact manifolds.

Definition 2.1. A contact structure on a manifold $M$ of dimension $2 n+1$ is a co-dimension one distribution $\xi$ on $M$ which is given locally by the kernel of a 1 -form $\alpha$ with $\alpha \wedge(d \alpha)^{n} \neq 0$. If there is a global 1 -form $\alpha$ with ker $\alpha=\xi$, then $\xi$ is called co-orientable, $\alpha$ is said to represent $\xi$, and is called a contact form.

Remark 2.2. One can define a co-orientable contact structure on a manifold $M$ in the following convenient way, which we exploit in a later section. A co-dimension one distribution $\xi$ is a co-orientable contact structure if its annihilator $\xi^{\circ}$ admits a nowhere vanishing section, $\alpha$, and $\xi^{\circ}-\{0\}$ is a symplectic submanifold of $T^{*} M$. Equivalently, a line sub-bundle $\zeta \subseteq T^{*} M$ which is symplectic away from the zero section and equipped with a nowhere vanishing section defines a co-orientable contact structure on $M$. A choice of a component of $\xi^{\circ}-\{0\}$ is a co-orientation for $\xi$

If $f$ is a non-vanishing function on $M$, then $f \alpha$ is another contact form on $M$ which represents $\xi$. The conformal class of $\alpha$ is

$$
\{f \alpha \mid f \text { is a positive function }\} \text {. }
$$

It should be stressed that the important data on a contact manifold is the contact structure, not the contact form used to represent it. Observe that for each $m \in M$,

$$
\left(\operatorname{ker} \alpha_{m}, d \alpha_{m \mid \operatorname{ker} \alpha_{m}}\right. \text { ) }
$$

is a symplectic vector space. On a co-oriented contact manifold $(M, \alpha)$ there is a distinguished vector field $Y$, called the Reeb vector field, which satisfies

$$
\iota(Y) \alpha=1, \quad \iota(Y) d \alpha=0 .
$$

$Y$ is unique with respect to these two properties. This allows us to split the tangent bundle of $M$ as

$$
T M=\operatorname{ker} \alpha \oplus \mathbb{R} Y
$$

where $\mathbb{R} Y$ is the line bundle over $M$ spanned by $Y$. Since $\mathcal{L}_{Y} \alpha=d \iota(Y) d \alpha+$ $d \iota(Y) \alpha=0$, the flow of the Reeb vector field preserves $\alpha$.

For the purposes of this article, the most important examples of contact manifolds come via interaction with symplectic manifolds.

Definition 2.3. A hypersurface $\Sigma$ of a symplectic manifold $(N, \omega)$ is said to be of contact type if there is a vector field $X$, defined near $\Sigma$, which is transverse to $\Sigma$ and satisfies $\mathcal{L}_{X} \omega=\omega . X$ is called a Liouville vector field. 
If $\Sigma$ is a hypersurface of contact type in a symplectic manifold $(N, \omega)$ with Liouville vector field $X$, then $\left(\Sigma, \iota(X) \omega_{\mid \Sigma}\right)$ is a contact manifold.

Example 2.4. If $(M, \xi)$ is a contact manifold, then away from the zero section the annihilator of $\xi$ in $T^{*} M$ is a symplectic submanifold of $T^{*} M$. A connected component of $\xi^{\circ}-\{0\}$ is called the symplectization of $(M, \xi)$. If $(M, \alpha)$ is a co-oriented contact manifold, then the symplectization can be identified with $\left(M \times \mathbb{R}, d\left(e^{t} \alpha\right)\right)$, where $t$ is the $\mathbb{R}$ coordinate. Note that every co-oriented contact manifold can be embedded as a hypersurface of contact type in its symplectization.

Definition 2.5. Suppose a Lie group $G$ acts on a contact manifold $(M, \alpha)$ and preserves $\alpha$. The contact moment map associated to the action is denoted by $\Phi_{\alpha}: M \rightarrow \mathfrak{g}^{*}$ and defined by

$$
\left\langle\Phi_{\alpha}(x), A\right\rangle=\alpha_{x}\left(A_{M}(x)\right)
$$

for $x \in M$ and all $A \in \mathfrak{g}$. The contact moment map is equivariant, where $G$ acts on $\mathfrak{g}^{*}$ through the co-adjoint respresentation $([\mathrm{Ge}])$.

Remark 2.6. The moment map depends upon the choice of an invariant contact form to represent the contact structure: if $f$ is a non-vanishing invariant function on $M$, then $f \alpha$ is an invariant contact form on $M$ and $\Phi_{f \alpha}=f \Phi_{\alpha}$.

Example 2.7. Suppose $(N, \omega)$ is a symplectic manifold equipped with a Hamiltonian $G$-action and let $\Psi: N \rightarrow \mathfrak{g}^{*}$ be a corresponding equivariant symplectic moment map. Let $\Sigma \subset N$ be an invariant hypersurface which has an invariant Liouville vector field and $\alpha$ the induced invariant contact form on $\Sigma$. Then the restriction of the $G$-action to $\Sigma$ preserves $\alpha$ and the associated contact moment map $\Phi_{\alpha}: \Sigma \rightarrow \mathfrak{g}^{*}$ is the restriction of $\Psi$ to $\Sigma$.

Example 2.8. Suppose a Lie group $G$ acts on a contact manifold $(M, \alpha)$ preserving $\alpha$ and let $\Phi_{\alpha}: M \rightarrow \mathfrak{g}^{*}$ be the associated contact moment map. The extension of the action to the symplectization $\left(M \times \mathbb{R}, d\left(e^{t} \alpha\right)\right)$ by $g \cdot(m, t)=(g \cdot m, t)$ is Hamiltonian and a corresponding equivariant symplectic moment map $\Psi: M \times \mathbb{R} \rightarrow \mathfrak{g}^{*}$ is given by $\Psi(m, t)=e^{t} \Phi_{\alpha}(m)$.

Lemma 2.9. Suppose a Lie group $G$ acts on a contact manifold $(M, \alpha)$, preserving $\alpha$, and let $\Phi_{\alpha}: M \rightarrow \mathfrak{g}^{*}$ be the associated moment map. Let $\Theta \subset \mathfrak{g}^{*}$ be a subset which is invariant under dilations by $\mathbb{R}^{+}$.

(1) Let $\Psi: M \times \mathbb{R} \rightarrow \mathfrak{g}^{*}$ be the symplectic moment map for the induced action on $M \times \mathbb{R}$. Then $\Psi^{-1}(\Theta)=\Phi_{\alpha}^{-1}(\Theta) \times \mathbb{R}$.

(2) If $f$ is a positive invariant function on $M$, then $\Phi_{f \alpha}^{-1}(\Theta)=\Phi_{\alpha}^{-1}(\Theta)$.

Proof. Because $\Psi(m, t)=e^{t} \Phi_{\alpha}(m)$ and $\Theta$ is $\mathbb{R}^{+}$invariant, $(m, t) \in \Psi^{-1}(\Theta)$ if and only if $\Phi_{\alpha}(m) \in \Theta$. This establishes the first point. The second one follows in the same manner, using the fact that $\Phi_{f \alpha}=f \Phi_{\alpha}$.

Recall that an action of a Lie group $G$ on a manifold $M$ is called proper if the map $G \times M \rightarrow M \times M$ given by $(g, m) \mapsto(g \cdot m, m)$ is a proper map. Actions of compact groups are proper. Proper actions have compact isotropy groups. If a Lie group $G$ acts properly on a (paracompact) co-oriented contact manifold $M$ and preserves the contact structure and a co-orientation, then we can find an invariant contact form, $\alpha$, on $M$ which represents the contact structure ([L1] ). In this case, the Reeb vector field is, by uniqueness, $G$-invariant as well. 
A slice for an action of a Lie group $G$ on a manifold $M$ at a point $x$ is a $G_{x}$ invariant submanifold $S$ such that $G \cdot S$ is an open subset of $M$ and such that the map $G \times S \rightarrow G \cdot S,(g, s) \mapsto g \cdot s$ descends to a diffeomorphism $G \times{ }_{G_{x}} S \rightarrow G \cdot S$, $[g, s] \mapsto g \cdot s$. A theorem of Palais asserts that for smooth proper actions slices exist at every point $([\overline{\mathrm{P}})$.

Proposition 2.10. Suppose a Lie group $G$ acts properly on a contact manifold $(M, \alpha)$, preserving $\alpha$, and let $\Phi_{\alpha}: M \rightarrow \mathfrak{g}^{*}$ be the associated contact moment map. If there exists a slice $S$ through $x \in \mathfrak{g}^{*}$ for the co-adjoint action which is invariant under dilations by $\mathbb{R}^{+}$, then $R:=\Phi_{\alpha}^{-1}(S)$ is a $G_{x}$ invariant contact submanifold of $(M, \alpha)$ and the contact moment map for the $G_{x}$ action on $R$ is given by the restriction of the $\Phi_{\alpha}$ to $R$ followed by the natural projection of $\mathfrak{g}^{*}$ onto $\mathfrak{g}_{x}^{*}$. $R$ is called a contact cross section.

Proof. The proof of Proposition 2.10 is derived from the corresponding proof of the symplectic cross section theorem of Guillemin and Sternberg ([LMTW]). Indeed, extend the $G$-action to the symplectization of $M$ and let $\Psi$ be the corresponding symplectic moment map. The symplectic cross section theorem gives $\Psi^{-1}(R)$ as a symplectic submanifold of the symplectization. By hypothesis, $S$ is invariant under dilations by $\mathbb{R}^{+}$and hence Lemma 2.9 implies that $\Psi^{-1}(R)=\Phi_{\alpha}^{-1}(R) \times \mathbb{R}$. It follows that the contact cross section is a hypersurface of contact type in the symplectic cross section, which gives the result.

Remark 2.11. Although Proposition 2.10 can be proved directly, the direct proof does not diverge significantly from the proof of the symplectic cross section theorem of Guillemin and Sternberg found in [LMTW].

\section{The REDUCTION THEOREMS}

In this section we prove the first two of our three main theorems.

Proposition 3.1. Suppose a Lie group $G$ acts properly on a contact manifold $(M, \alpha)$, preserving $\alpha$ and let $\Phi_{\alpha}: M \rightarrow \mathfrak{g}^{*}$ be the associated moment map.

1. The Reeb flow preserves the level sets of $\Phi_{\alpha}$.

2. For all $x \in M, \vec{v} \in T_{x} M$, and $A \in \mathfrak{g}$,

$$
\left\langle d\left(\Phi_{\alpha}\right)_{x}(\vec{v}), A\right\rangle=d \alpha_{m}\left(\vec{v}, A_{M}(x)\right) .
$$

3. If $\Phi_{\alpha}(x)=0$, then $T_{x}(G \cdot x)$ is an isotropic subspace of the symplectic vector space $\left(\operatorname{ker} \alpha_{x}, d \alpha_{x}\right)$.

4. $\operatorname{Im}\left(d\left(\Phi_{\alpha}\right)_{x}\right)^{\circ}=\left\{A \in \mathfrak{g} \mid \iota\left(A_{M}(x)\right) d \alpha_{x}=0\right\}=\left\{A \in \mathfrak{g} \mid A_{M}(x) \in\right.$ ker $\left.d \alpha_{x}\right\}$.

Proof. Denote the Reeb vector field by $Y$ and its flow by $\rho_{t}$. As noted earlier, by uniqueness $Y$ is invariant and therefore $\rho_{t}$ is equivariant. Therefore, for any $A \in \mathfrak{g}$ and $m \in M$, it follows that $A_{M}\left(\rho_{t}(m)\right)=d \rho_{t}\left(A_{M}(m)\right)$. Thus

$$
\begin{aligned}
\left\langle\Phi_{\alpha}\left(\rho_{t}(z)\right), A\right\rangle & =\alpha_{\rho_{t}(z)}\left(A_{M}\left(\rho_{t}(z)\right)\right) \\
& =\rho_{t}^{*}(\alpha)_{z}\left(A_{M}(z)\right) \\
& =\alpha_{z}\left(A_{M}(z)\right) \\
& =\left\langle\Phi_{\alpha}(z), A\right\rangle .
\end{aligned}
$$

This establishes item (1). 
Since $\mathcal{L}_{A_{M}} \alpha=0$, Cartan's formula gives $d \iota\left(A_{M}\right) \alpha=-d \alpha\left(A_{M},-\right)$. Therefore,

$$
\left\langle d\left(\Phi_{\alpha}\right)_{x}(\vec{v}), A\right\rangle=d\left\langle\Phi_{\alpha}, A\right\rangle_{x}(\vec{v})=d \alpha_{x}\left(\vec{v}, A_{M}(x)\right),
$$

which establishes item (2).

The proof of item (3) is an application of item (2). For any $A, B \in \mathfrak{g}$, the second item implies that for any $x \in \Phi_{\alpha}^{-1}(0)$,

$$
\begin{aligned}
d \alpha_{x}\left(A_{M}(x), B_{M}(x)\right) & =\left\langle d\left(\Phi_{\alpha}\right)_{x}\left(A_{M}(x)\right), B\right\rangle \\
& =\left\langle A_{\mathfrak{g}^{*}}\left(\Phi_{\alpha}(x)\right), B\right\rangle \quad \text { by equivariance } \\
& =\left\langle A_{\mathfrak{g}^{*}}(0), B\right\rangle \\
& =0 .
\end{aligned}
$$

Thus, $A \in T_{x}(G \cdot x)^{d \alpha_{x}}$, whence $T_{x}(G \cdot x) \subseteq T_{x}(G \cdot x)^{d \alpha_{x}}$.

The final point follows immediately from the second point. Note that ker $d \alpha_{x}=$ $\mathbb{R} Y(x)$.

Remark 3.2. Note that the last item of Proposition 3.1 implies that $x \in M$ is a regular point for $\Phi$ if and only if

$$
\operatorname{dim}\left\{A \in \mathfrak{g} \mid A_{M}(x) \in \mathbb{R} Y(x)\right\}=0 .
$$

That is, $x \in M$ may be critical yet have a discrete stabilizer. This contrasts sharply with the symplectic category, where a point is regular if and only if its stabilizer is discrete.

Lemma 3.3. Let $G$ be a Lie group and choose $\mu \in \mathfrak{g}^{*}$. Then $\mathfrak{k}_{\mu}=\operatorname{ker} \mu \cap \mathfrak{g}^{*}=$ $\operatorname{ker}\left(\mu_{\mid \mathfrak{g}_{\mu}}\right)$ is a Lie ideal in $\mathfrak{g}_{\mu}$. Hence, there is a unique, connected normal Lie subgroup of $G_{\mu}$ with Lie algebra $\mathfrak{k}_{\mu}$.

Proof. Choose $A \in \mathfrak{g}_{\mu}$. Then $0=A_{\mathfrak{g}^{*}}(\mu)=\operatorname{ad}^{\dagger}(A) \mu$, where $\operatorname{ad}^{\dagger}: \mathfrak{g} \rightarrow \operatorname{End}\left(\mathfrak{g}^{*}\right)$ is the differential of the co-adjoint representation. Thus, for any $B \in \operatorname{ker}\left(\mu_{\mid \mathfrak{g}_{\mu}}\right)$, we have that

$$
0=\left\langle\operatorname{ad}^{\dagger}(A) \mu, B\right\rangle=-\langle\mu,[A, B]\rangle
$$

Therefore, $\mathfrak{k}_{\mu}$ is a Lie ideal in $\mathfrak{g}_{\mu}$.

Definition 3.4. Suppose a Lie group $G$ acts properly on a contact manifold, $(M, \alpha)$, preserving $\alpha$, and let $\Phi_{\alpha}: M \rightarrow \mathfrak{g}^{*}$ be the associated moment map. Choose $\mu \in \mathfrak{g}^{*}$. We define the kernel group of $\mu$ to be the unique connected Lie subgroup of $G_{\mu}$ with Lie algebra $\mathfrak{k}_{\mu}:=\operatorname{ker} \mu_{\mid \mathfrak{g}_{\mu}}$. The kernel group of $\mu$ is denoted by $K_{\mu}$. We define the contact quotient (or contact reduction) of $M$ by $G$ at $\mu$ to be

$$
M_{\mu}:=\Phi_{\alpha}^{-1}\left(\mathbb{R}^{+} \mu\right) / K_{\mu} .
$$

Remark 3.5. If $f$ is a positive invariant function on $M$, then $f \alpha$ is another invariant contact form on $M$ which is in the same conformal class as $\alpha$. Since $\mathbb{R}^{+} \mu$ is invariant under dilations by $\mathbb{R}^{+}$, Lemma 2.9 implies that $\Phi_{f \alpha}^{-1}\left(\mathbb{R}^{+} \mu\right)=\Phi_{\alpha}^{-1}\left(\mathbb{R}^{+} \mu\right)$ and hence the reduced space is topologically independent of the choice of contact forms in the same conformal class.

Lemma 3.6. Suppose a Lie group $G$ acts on a contact manifold $(M, \alpha)$, preserving $\alpha$, and let $\Phi_{\alpha}: M \rightarrow \mathfrak{g}^{*}$ be the associated contact moment map. Choose $\mu \in \mathfrak{g}^{*}$ and let $K_{\mu}$ be the connected Lie subgroup of $G_{\mu}$ with Lie algebra $\mathfrak{k}_{\mu}=\operatorname{ker} \mu_{\mid \mathfrak{g}_{\mu}}$. Then $\Phi_{\alpha}$ is transverse to $\mathbb{R}^{+} \mu$ if and only if $K_{\mu}$ acts locally freely on $\Phi_{\alpha}^{-1}\left(\mathbb{R}^{+} \mu\right)$. 
Proof. If $\Phi_{\alpha}$ is transverse to $\mathbb{R}^{+} \mu$, then

$$
Z:=\Phi_{\alpha}^{-1}\left(\mathbb{R}^{+} \mu\right)
$$

is a submanifold of $M$. Choose $z \in Z$. The transversality condition,

$$
\operatorname{Im}\left(d\left(\Phi_{\alpha}\right)_{z}\right)+\mathbb{R} \mu=\mathfrak{g}^{*},
$$

is equivalent to the condition

$$
\operatorname{Im}\left(d\left(\Phi_{\alpha}\right)_{z}\right)^{\circ} \cap \operatorname{ker} \mu=0 .
$$

Let $H$ be the isotropy subgroup of $z$ in $K_{\mu}$ and $\mathfrak{h}$ its Lie algebra. Choose $A \in \mathfrak{h}$ and denote the Reeb vector field of $(M, \alpha)$ by $Y$. Then $\langle\mu, A\rangle=0$ since $\mathfrak{h} \subseteq \mathfrak{k}_{\mu}$. Because $H$ fixes $z, A_{M}(z)=0$. Thus,

$$
\begin{aligned}
A & \in\left\{B \in \mathfrak{g} \mid B_{M}(z) \in \mathbb{R} Y(z)\right\} \cap \operatorname{ker} \mu \\
& =\operatorname{Im}\left(d\left(\Phi_{\alpha}\right)_{z}\right)^{\circ} \cap \operatorname{ker} \mu \quad \text { by Proposition } 3.1 \\
& =0 .
\end{aligned}
$$

Therefore, $\mathfrak{h}=0$ and $K_{\mu}$ acts locally freely on $Z$.

Conversely, suppose that $\Phi_{\alpha}(z)=s \mu$ for some $s \in \mathbb{R}^{+}$and let $A \in \operatorname{ker} \mu \cap$ $\left(\operatorname{Im}\left(d\left(\Phi_{\alpha}\right)_{z}\right)\right)^{\circ}$. Then, by Proposition 3.1, $A_{M}(z)=t Y(z)$ for some $t \in \mathbb{R}$, where $Y$ is the Reeb vector field. Therefore,

$$
0=\langle s \mu, A\rangle=\left\langle\Phi_{\alpha}(z), A\right\rangle=\alpha_{z}\left(A_{M}(z)\right)=\alpha_{z}(t Y(z))=t .
$$

Hence, $A_{M}(z)=0$ and $A \in \mathfrak{g}_{z}$. Since $A \in$ ker $\mu$ and $A \in \mathfrak{g}_{z} \subseteq \mathfrak{g}_{\mu}$, it follows that $A \in \mathfrak{k}_{\mu}$. Since $K_{\mu}$ acts locally freely on $\Phi_{\alpha}^{-1}\left(\mathbb{R}^{+} \mu\right), A=0$ and hence $\Phi_{\alpha}$ is transverse to $\mathbb{R}^{+} \mu$.

While the contact quotient is defined at any element of $\mathfrak{g}^{*}$, it is not necessarily a contact manifold. There are two problems. The first is that there is no guarantee that the kernel group of $\mu$ will act properly on $\Phi^{-1}\left(\mathbb{R}^{+} \mu\right)$, in which case the resulting quotient may not be Hausdorff. If $G$ is compact and $\mu$ is integral (i.e., $K_{\mu}=\operatorname{ker} \chi_{\mu}$, where $\chi_{\mu}: G_{\mu} \rightarrow S^{1}$ is a group map satisfying $\left.d \chi_{u}=\mu_{\mid \mathfrak{g}_{\mu}}\right)$, then $K_{\mu}$ is actually compact and no hypothesis is needed. This integrality condition is required in the Guillemin-Sternberg procedure, described later (GS1]). The second problem is that the kernel and isotropy groups of $\mu$ may coincide. If $\mu$ is non-zero, then the resulting quotient may fail to be contact, as the below example shows. Hence, we assume that ker $\mu+\mathfrak{g}_{\mu}=\mathfrak{g}$. If $G$ is compact, then the existence of an invariant metric on $G$ implies this condition.

Example 3.7. Let $G=\operatorname{SL}(2, \mathbb{R})$ and let $\alpha$ be the natural contact form on $M=$ $T^{*}(G) \times \mathbb{R} \cong G \times \mathfrak{g}^{*} \times \mathbb{R}$. For the lift of left multiplication the associated contact moment map, $\Phi_{\alpha}: M \rightarrow \mathfrak{g}^{*}$, is given by $\Phi_{\alpha}(g, \vec{v}, t)=\operatorname{Ad}^{\dagger}(g) \cdot \vec{v}$. Set

$$
\mu=\left(\begin{array}{ll}
0 & 1 \\
0 & 0
\end{array}\right) \text {. }
$$

Then

$$
\mathfrak{k}_{\mu}=\mathfrak{g}_{\mu}=\left\{\left(\begin{array}{cc}
0 & t \\
0 & 0
\end{array}\right) \mid t \in \mathbb{R}\right\}
$$

Therefore,

$$
K_{\mu}=\left\{\left(\begin{array}{cc}
1 & t \\
0 & 1
\end{array}\right) \mid t \in \mathbb{R}\right\}
$$


is closed in $G$ and acts properly on $M$, but $\Phi_{\alpha}^{-1}\left(\mathbb{R}^{+} \mu\right) / K_{\mu}$ is four dimensional and hence is not contact.

Lemma 3.8. Let $V$ be a vector space and $\omega: V \times V \rightarrow \mathbb{R}$ be an antisymmetric bilinear map. Suppose there is a decomposition $V=X \oplus W$ which is perpendicular with respect to $\omega$; i.e., $\omega(x, w)=0$ for all $x \in X$ and $w \in W$. If $\operatorname{ker} \omega \subseteq \operatorname{ker} \omega_{\mid X}$, then $\operatorname{ker} \omega=\operatorname{ker} \omega_{\mid X}$.

Proof. Choose $x_{0} \in \operatorname{ker} \omega_{\mid X}$ and $v \in V$. Write $v=x+w$ where $x \in X$ and $w \in W$. Then $\omega\left(x_{0}, v\right)=\omega\left(x_{0}, x\right)+\omega\left(x_{0}, w\right)=0$. Hence $x_{0} \in \operatorname{ker} \omega$.

Lemma 3.9. Let $(V, \omega)$ be a symplectic vector space and suppose that $W$ is an isotropic subspace. Then

$$
\operatorname{ker} \omega_{\mid W^{\omega}}=W
$$

where $W^{\omega}$ is the symplectic perpendicular of $W$ with respect to $\omega$.

Proof. Fix $w \in W$. Since $W$ is isotropic, $w \in W^{\omega}$. For any $x \in W^{\omega}, \omega(w, x)=0$ by definition. Hence $W \subseteq \operatorname{ker} \omega_{\mid W^{\omega}}$. Conversely, if $x \in \operatorname{ker} \omega_{W^{\omega}}$, then by unravelling the various definitions, one sees that $x \in\left(W^{\omega}\right)^{\omega}=W$.

Theorem 1. Suppose a Lie group $G$ acts on a contact manifold, $(M, \alpha)$, preserving $\alpha$, and let $\Phi_{\alpha}: M \rightarrow \mathfrak{g}^{*}$ be the associated contact moment map. Choose $\mu \in \mathfrak{g}^{*}$ and let $K_{\mu}$ be the connected Lie subgroup of $G_{\mu}$ with Lie algebra $\mathfrak{k}_{\mu}=\operatorname{ker} \mu_{\mid \mathfrak{g}_{\mu}}$. If

- $K_{\mu}$ acts properly on $\Phi_{\alpha}^{-1}\left(\mathbb{R}^{+} \mu\right)$,

- $\Phi$ is transverse to $\mathbb{R}^{+} \mu$,

- $\operatorname{ker} \mu+\mathfrak{g}_{\mu}=\mathfrak{g}$,

then the quotient

$$
M_{\mu}=\Phi_{\alpha}^{-1}\left(\mathbb{R}^{+} \mu\right) / K_{\mu}
$$

is naturally a contact orbifold; i.e.,

$$
\text { ker } \alpha \cap T\left(\Phi_{\alpha}^{-1}\left(\mathbb{R}^{+} \mu\right)\right)
$$

descends to a contact structure on the quotient $M_{\mu}$.

Remark 3.10. In the special case of $\mu=0$, C. Albert, H. Geiges, and F. Loose, independently of Guillemin-Sternberg, established the above theorem in various papers ( $\mathrm{Al}, \mathrm{Ge}, \mathrm{LO})$.

Proof. Since $\Phi_{\alpha}$ is transverse to $\mathbb{R}^{+} \mu, Z:=\Phi_{\alpha}^{-1}\left(\mathbb{R}^{+} \mu\right)$ is a submanifold of $M$ and Lemma 3.6 implies that $K_{\mu}$ acts locally freely on $Z$. Hence, $M_{\mu}$ is an orbifold. Fix $z \in Z$. For any $A \in \mathfrak{k}_{\mu}$, we have that

$$
\begin{aligned}
\left(\iota\left(A_{M}\right) \alpha\right)(z) & =\left\langle\Phi_{\alpha}(z), A\right\rangle \\
& =\langle s \mu, A\rangle \quad \text { for some } s \in \mathbb{R}^{+} \\
& =0 .
\end{aligned}
$$

Hence, $\alpha$ descends to $\alpha_{\mu}$ on $M_{\mu}$, which is contact if and only if

$$
\operatorname{ker}\left(d \alpha_{z \mid T_{z} Z \cap \operatorname{ker} \alpha_{z}}\right)=T_{z}\left(K_{\mu} \cdot z\right) \text {. }
$$

Let $\Psi_{\alpha}: M \rightarrow \mathfrak{k}_{\mu}^{*}$ be the contact moment map associated to the action of $K_{\mu}$ on $M$. Note that $\Psi_{\alpha}=i^{T} \circ \Phi_{\alpha}$ where $i^{T}: \mathfrak{g}^{*} \rightarrow \mathfrak{k}_{\mu}^{*}$ is the natural projection. Observe that $\Psi_{\alpha}(z)=0$ and hence that $T_{z}\left(K_{\mu} \cdot z\right)$ is an isotropic subspace of the symplectic vector space $\left(\operatorname{ker} \alpha_{z}, d \alpha_{z \mid \operatorname{ker} \alpha_{z}}\right.$ ) by Proposition 3.1 Let $T_{z}\left(K_{\mu} \cdot z\right)^{d \alpha_{z}}$ 
denote the symplectic perpendicular of $T_{z}\left(K_{\mu} \cdot z\right)$ in $\left(\operatorname{ker} \alpha_{z}, d \alpha_{z \mid \operatorname{ker} \alpha_{z}}\right)$. A vector $\vec{v}$ is in $T_{z}\left(K_{\mu} \cdot z\right)^{d \alpha_{z}}$ if and only if for all $A \in \mathfrak{k}_{\mu}$,

$$
\begin{aligned}
0 & =d \alpha_{z}\left(\vec{v}, A_{M}(z)\right) \\
& =\left\langle d\left(\Phi_{\alpha}\right)_{z}(\vec{v}), A\right\rangle \quad \text { by Proposition 3.1. }
\end{aligned}
$$

That is, $\vec{v} \in T_{z}\left(K_{\mu} \cdot z\right)^{d \alpha_{z}}$ if and only if $d\left(\Phi_{\alpha}\right)_{z}(\vec{v})_{\mid \mathfrak{k}_{\mu}} \equiv 0$. Hence,

$$
T_{z}\left(K_{\mu} \cdot z\right)^{d \alpha_{z}}=T_{z} U \cap \operatorname{ker} \alpha_{z}
$$

where $U=\Phi_{\alpha}^{-1}\left(\mathfrak{k}_{\mu}^{\circ}\right)$. Note that $U$ is a submanifold of $M$ by the transversality condition. Indeed, $U=\Phi_{\alpha}^{-1}\left(\mathfrak{k}_{\mu}^{\circ}\right)=\Psi_{\alpha}^{-1}(0)$. Since $\Phi_{\alpha}$ is transverse to $\mathbb{R}^{+} \mu, K_{\mu}$ acts locally freely on $Z$ by Lemma 3.6 and thus on a neighborhood of $Z$ and hence on $U$ (or, at least on a neighborhood of $Z$ in $U$ ). Therefore, also by Lemma [3.6, $U$ is a submanifold of $M$. Lemma 3.9$]$ implies that $T_{z}\left(K_{\mu} \cdot z\right)=\operatorname{ker}\left(d \alpha_{z \mid T_{z} U \cap \operatorname{ker} \alpha_{z}}\right)$.

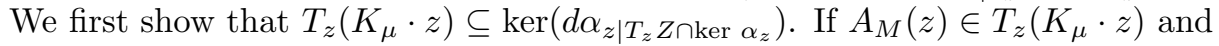
$\vec{v} \in T_{z} Z \cap \operatorname{ker} \alpha_{z}$, then

$$
\begin{aligned}
d \alpha_{z}\left(A_{M}(z), \vec{v}\right) & =-\left\langle d\left(\Phi_{\alpha}\right)_{z}(\vec{v}), A\right\rangle \\
& =s\langle\mu, A\rangle \\
& =0
\end{aligned}
$$

Therefore,

$$
T_{z}\left(K_{\mu} \cdot z\right) \subseteq \operatorname{ker}\left(d \alpha_{\left.z \mid T_{z} Z \cap \operatorname{ker} \alpha_{z}\right)}\right.
$$

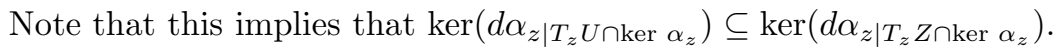

The reverse inclusion is slightly more delicate. Since ker $\mu+\mathfrak{g}_{\mu}=\mathfrak{g}$, we can choose a splitting $\mathfrak{g}=\mathfrak{g}_{\mu} \oplus \mathfrak{m}$ where $\mu_{\mid \mathfrak{m}} \equiv 0$. Let $\mathfrak{m}_{M}(z)=\left\{A_{M}(z) \mid A \in \mathfrak{m}\right\}$. The proof is completed by showing that $\mathfrak{m}_{M}(z)$ and $T_{z} Z \cap$ ker $\alpha_{z}$ are complementary subspaces of $T_{z} U \cap$ ker $\alpha_{z}$ which are prependicular with respect to $d \alpha_{z \mid T_{z} U \cap \operatorname{ker} \alpha_{z}}$. Lemma 3.8 implies the reverse inclusion.

We first show that $\mathfrak{m}_{M}(z) \subset T_{z} U \cap$ ker $\alpha_{z}$. Choose any $A \in \mathfrak{m}$ and let $B \in \mathfrak{k}_{\mu}$. Then for some $t \in \mathbb{R}^{+}$,

$$
\begin{aligned}
\left\langle d\left(\Phi_{\alpha}\right)\left(A_{M}(z)\right), B\right\rangle & =\left\langle A_{\mathfrak{g}^{*}}(t \mu), B\right\rangle \quad \text { by equivariance } \\
& =t\langle\mu,[A, B]\rangle \\
& =-t\left\langle B_{\mathfrak{g}^{*}}(\mu), A\right\rangle \\
& =0
\end{aligned}
$$

since $B \in \mathfrak{k}_{\mu} \subset \mathfrak{g}_{\mu}$. Hence, $\mathfrak{m}_{M}(z) \subset T_{z} U$. Additionally,

$$
\begin{aligned}
\alpha_{z}\left(A_{M}(z)\right) & =\left\langle\Phi_{\alpha}(z), A\right\rangle \\
& =t\langle\mu, A\rangle \quad \text { for some } t \in \mathbb{R}^{+} \\
& =0 \quad \text { since } \mu_{\mid \mathfrak{m}} \equiv 0 .
\end{aligned}
$$

Thus, $\mathfrak{m}_{M}(z) \subset T_{z} U \cap$ ker $\alpha_{z}$.

By equivariance, $d\left(\Phi_{\alpha}\right)_{z}\left(A_{M}(z)\right)=A_{\mathfrak{g}}^{*}(t \mu)$. Hence, $d\left(\Phi_{\alpha}\right)_{z}$ maps $\mathfrak{m}_{M}(z)$ to $T_{t \mu}(G \cdot t \mu)$. In fact, one can show that this map is an isomorphism. By definition, $d\left(\Phi_{\alpha}\right)_{z}\left(T_{z} Z\right)=\mathbb{R} \mu$. The assumption ker $\mu+\mathfrak{g}_{\mu}=\mathfrak{g}$ is equivalent to

$$
\begin{aligned}
0 & =(\operatorname{ker} \mu)^{\circ} \cap\left(\mathfrak{g}_{\mu}\right)^{\circ} \\
& =\mathbb{R} \mu \cap T_{t \mu}(G \cdot t \mu) .
\end{aligned}
$$


Hence, $\mathfrak{m}_{M}(z) \cap T_{z} Z=\{0\}$. Finally, if $A_{M}(z)=0$, then $A \in \mathfrak{g}_{z} \subseteq \mathfrak{m}_{\mu}$. Hence, $\mathfrak{m}_{M}(z)$ is a subspace of dimension $\operatorname{dim} \mathfrak{m}=\operatorname{dim} \mathfrak{g}-\operatorname{dim} \mathfrak{g}_{\mu}$. A dimension count implies that $\mathfrak{m}_{M}(z)$ and $T_{z} Z \cap \operatorname{ker} \alpha_{z}$ are complementary subspaces of $T_{z} U \cap \operatorname{ker} \alpha_{z}$, which completes the proof.

The second main theorem removes the transversality condition above but replaces it with a requirement that a convex, $\mathbb{R}^{+}$-invariant slice exists for $\mu \in \mathfrak{g}^{*}$. This condition is satisfied for compact $G$. The author suspects, but cannot prove, that a stratification theorem for proper group actions ought to hold. In the particular case of $\mu=0$, the author and E. Lerman showed that $M_{0}$ is topologically a stratified space:

Theorem 3.11. Let $M$ be a manifold with a co-oriented contact structure $\xi$. Suppose a Lie group $G$ acts properly on $M$ preserving $\xi$ and a co-orientation for $\xi$. Choose a $G$-invariant contact form $\alpha$ with $\operatorname{ker} \alpha=\xi$ and let $\Phi_{\alpha}: M \rightarrow \mathfrak{g}^{*}$ be the corresponding moment map.

Then for every subgroup $H$ of $G$, each connected component of the topological space

$$
\left(M_{(H)} \cap \Phi_{\alpha}^{-1}(0)\right) / G
$$

is a manifold and the partition of the contact quotient

$$
M_{0} \equiv M / / G:=\Phi_{\alpha}^{-1}(0) / G
$$

into these manifolds is a stratification. The symbol $M_{(H)}$ stands for the set of points in $M$ with the isotropy groups conjugate to $H$.

Proof. See $\mathrm{LW}$ for a complete proof.

Proposition 3.12. Suppose a Lie group $G$ acts on a contact manifold $(M, \alpha)$, preserving $\alpha$, and let $\Phi_{\alpha}: M \rightarrow \mathfrak{g}^{*}$ be the associated contact moment map. Choose $\mu \in \mathfrak{g}^{*}$. Suppose

- The kernel group $K_{\mu}$ of $\mu$ acts properly on $R:=\Phi_{\alpha}^{-1}(S)$.

- There is a convex slice $S$ for $\mu$ which is invariant under dilations by $\mathbb{R}^{+}$.

Denote the natural projection, $\mathfrak{g}^{*} \rightarrow \mathfrak{g}_{\mu}^{*}$ by $i^{T}$ and let $\mu^{\prime}=i^{T}(\mu)$. Then $M_{\mu}=R_{\mu^{\prime}}$, where $R_{\mu^{\prime}}$ is the reduction of $R$ by $G_{\mu}$ at $\mu^{\prime}$.

Proof. By Proposition 2.10 the restriction of $\alpha$ to $R$ is a contact form and $R$ is $G_{\mu}$ invariant. The contact moment map for the $G_{\mu}$-action on $R$ is given by $\Psi_{\alpha}=$ $i^{T} \circ \Phi_{\alpha \mid R}$. Note that

$$
\Psi_{\alpha}^{-1}\left(\mathbb{R}^{+} \mu^{\prime}\right)=\Phi_{\alpha}^{-1}\left(\mathbb{R}^{+} \mu+\mathfrak{g}_{\mu}^{\circ}\right) .
$$

The proof will be completed by showing that $\left(\mathbb{R}^{+} \mu+\mathfrak{g}_{\mu}^{\circ}\right) \cap S=\mathbb{R}^{+} \mu$. From this it follows that $\Phi_{\alpha}^{-1}\left(\mathbb{R}^{+} \mu\right)=\Psi_{\alpha}^{-1}\left(\mathbb{R}^{+} \mu^{\prime}\right)$. Since $K_{\mu}=K_{\mu^{\prime}}$, the conclusion follows.

Since $S$ is $\mathbb{R}^{+}$-invariant and contains $\mu, \mathbb{R}^{+} \mu \subseteq\left(\mathbb{R}^{+} \mu+\mathfrak{g}_{\mu}^{\circ}\right) \cap S$. Recall that $\mathfrak{g}_{\mu}^{\circ}=T_{\mu}(G \cdot \mu)$. If $t \mu+\eta \in\left(\mathbb{R}^{+} \mu+\mathfrak{g}_{\mu}^{\circ}\right) \cap S$, then $\gamma(s)=\mu+\frac{s}{t} \eta, s \in[0,1]$ is tangent to $G \cdot \mu$ at $\mu$. On the other hand, since $\gamma(0), \gamma(1) \in S$ and $S$ is convex, it follows that $\gamma$ is also tangent to $S$ at $\mu$. Hence $\gamma \in T_{\mu}(G \cdot \mu) \cap T_{\mu} S$. However, because $S$ is a slice, $T_{\mu}(G \cdot \mu) \cap T_{\mu} S=0$. Thus, $\eta=0$ and $\left(\mathbb{R}^{+} \mu+\mathfrak{g}_{\mu}^{\circ}\right) \cap S=\mathbb{R}^{+} \mu$.

Remark 3.13. Proposition 3.12 allows us to always assume that, given that the hypotheses are satisfied, the element at which we are reducing is always fixed by the co-adjoint action of the symmetry group. The hypotheses of Proposition 3.12 
are satisifed for the action of any compact group. Indeed, if $G$ is compact, we can choose a $G$-invariant metric and hence an equivariant splitting $\mathfrak{g}^{*}=\mathfrak{g}_{\mu}^{\circ} \oplus \mathfrak{g}_{\mu}^{*}$, where $\mathfrak{g}_{\mu}^{*}$ is embedded as the normal fiber to $G \cdot \mu$ at $\mu$. Take $S=\mathbb{R}^{+} D_{\epsilon}$ where $D_{\epsilon}$ is a small $\epsilon$-ball about 0 in $\mathfrak{g}_{\mu}^{*}$. Then $S$ is a convex, $R^{+}$-invariant slice at $\mu$.

We can now prove our second main theorem.

Theorem 2. Suppose a Lie group $G$ acts on a contact manifold $(M, \alpha)$, preserving $\alpha$ and let $\Phi_{\alpha}: M \rightarrow \mathfrak{g}^{*}$ be the associated contact moment map. Choose $\mu \in \mathfrak{g}^{*}$ and let $K_{\mu}$ be the connected Lie subgroup of $G_{\mu}$ with Lie algebra $\mathfrak{k}_{\mu}=\operatorname{ker} \mu_{\mid \mathfrak{g}_{\mu}}$. Suppose

- $K_{\mu}$ acts properly on $\Phi_{\alpha}^{-1}\left(\mathbb{R}^{+} \mu\right)$;

- a convex slice exists for $\mu$ which is invariant under dilations by $\mathbb{R}^{+}$.

Then the partition of the contact quotient by $G$ at $\mu$ by orbit types,

$$
M_{\mu}=\coprod_{(H)} \frac{\Phi_{\alpha}^{-1}\left(\mathbb{R}^{+} \mu\right) \cap M_{(H)}}{K_{\mu}},
$$

is a stratification. Here $M_{(H)}$ is the set of points whose stabilizer is conjugate to $H$ and the indexing set is the set of conjugacy classes of stabilizer subgroups of $K_{\mu}$.

Proof. By Proposition 3.12, we may assume that $\mu$ is fixed by the co-adjoint action of $G$ on $\mathfrak{g}^{*}$. Let $\Psi_{\alpha}: M \rightarrow \mathfrak{k}_{\mu}^{*}$ be the moment map for the action of $K_{\mu}$. Because $\Psi_{\alpha}=i^{T} \circ \Phi_{\alpha}$, where $i^{T}: \mathfrak{g}^{*} \rightarrow \mathfrak{k}_{\mu}^{*}$ is the natural projection, we have $\Psi_{\alpha}^{-1}(0)=\Phi_{\alpha}^{-1}(\mathbb{R} \mu)$. Therefore, $M_{\mu}$ is an open subset of $M / / K_{\mu}$, which is stratified by Theorem 3.11. Open subsets of stratified spaces are naturally stratified.

\section{Contact structures on the strata of the Reduced space}

The previous section established the topological structure of the contact quotient but did not address the geometrical structure of the quotient under stratification. The point of this section is to show that the contact quotient is a contact stratified space; i.e., there exists a line bundle over $M_{\mu}$ which, when restricted to each stratum, defines a co-oriented contact structure.

Proposition 4.1. Suppose a Lie group G acts properly on a contact manifold $(M, \alpha)$, preserving $\alpha$, and let $\Phi_{\alpha}: M \rightarrow \mathfrak{g}^{*}$ be the associated moment map. Let $H$ be an isotropy subgroup of $G$,

$$
N=N(H)=\left\{g \in G \mid g H g^{-1}=H\right\}
$$

the normalizer of $H$ in $G, L=N(H) / H$, and

$$
M_{H}=\left\{m \in M \mid G_{m}=H\right\}
$$

the set of points of $M$ whose isotropy group is $H$. Then $M_{H}$ is a contact submanifold of $M, L$ acts freely on $M_{H}$, and there is a diffeomorphism,

$$
\vartheta: M_{H} / / L \rightarrow\left(\Phi_{\alpha}^{-1}(0) \cap M_{(H)}\right) / G \text {. }
$$

Proof. $M_{H}$ is a submanifold of $M$ and for all $x \in M_{H}$,

$$
T_{x}\left(M_{H}\right)=\left(T_{x} M\right)^{H}
$$

where $\left(T_{x} M\right)^{H}$ is the set of $H$-fixed vectors in $T_{x} M$ (see, for example, Proposition 27.5 of [GS2]). The Reeb vector field, $Y$, of $(M, \alpha)$, is $G$-invariant and, since the $H$-action preserves the contact structure,

$$
\left(T_{x} M\right)^{H}=\left(\operatorname{ker} \alpha_{z} \oplus \mathbb{R} Y(x)\right)^{H}=\left(\operatorname{ker} \alpha_{x}\right)^{H} \oplus \mathbb{R} Y(x) .
$$


Because $\left(\operatorname{ker} \alpha_{x}\right)^{H}$ is a symplectic subspace of $\left(\operatorname{ker} \alpha_{x}, d \alpha_{x \mid \operatorname{ker} \alpha_{x}}\right)$, the restriction of $\alpha$ to $M_{H}$ is a contact form on $M_{H}$.

In what follows it is useful to cite Lemma 17, pg. 220, of [BL], which identifies $\mathfrak{l}^{*}$, the dual of the Lie algebra of $L$, with $\left(\mathfrak{h}^{\circ}\right)^{H}$. For any $X \in \mathfrak{h}$ and $x \in M_{H}$,

$$
\left\langle\Phi_{\alpha}(x), X\right\rangle=\alpha_{x}\left(X_{M}(x)\right)=0
$$

since $X_{M}(x)=0$. Hence, the image of $M_{H}$ in $\mathfrak{g}^{*}$ under $\Phi_{\alpha}$ is contained in $\mathfrak{h}^{\circ}$. Because the moment map is equivariant, $\Phi_{\alpha}\left(M_{H}\right) \subseteq\left(\mathfrak{h}^{\circ}\right)^{H}$. The action of $L$ on $M_{H}$ is defined by $n H \cdot x=n \cdot x$, where $n H$ is the coset containing $n \in N$. This is free by definition. The moment map, $\Psi_{\alpha}: M_{H} \rightarrow \mathfrak{l}^{*}$, for the $L$-action on $M_{H}$ is given by the restriction of $\Phi_{\alpha}$ to $M_{H}$. Therefore,

$$
M_{H} / / L=\left(\Phi_{\alpha}^{-1}(0) \cap M_{H}\right) / L .
$$

The natural inclusion,

$$
\Phi_{\alpha}^{-1}(0) \cap M_{H} \rightarrow \Phi_{\alpha}^{-1}(0) \cap M_{(H)},
$$

descends to a map,

$$
\vartheta: M_{H} / / L \rightarrow\left(\Phi_{\alpha}^{-1}(0) \cap M_{(H)}\right) / G
$$

defined by $[x]_{L} \mapsto[x]_{G}$, where $[x]_{L}$ and $[x]_{G}$ denote the orbit through $x$ under the $L$ and $G$ actions respectively. If $x \in \Phi_{\alpha}^{-1}(0) \cap M_{(H)}$, then the stablilizer of $x$ in $G$ is conjugate to $H$. This implies that some element of the $G$-orbit through $x$ has stabilizer equal to $H$, whence $\vartheta$ is surjective.

To show that $\vartheta$ is injective, suppose that $x, y \in \Phi_{\alpha}^{-1}(0) \cap M_{H}$ and that $y=g \cdot x$. Because $x$ and $y$ have stabilizer equal to $H$, it follows that $g \in N$ and therefore that $\vartheta$ is injective.

By expressing each stratum of the contact quotient as the reduction at zero of a contact manifold by a freely acting symmetry group, we obtain a contact structure on each stratum. It is slightly less clear, however, how these structures are related to one another.

Remark 4.2. Suppose a Lie group $G$ acts on a manifold $N$ and $\sigma$ is an invariant 1 -form on $N$. Then $\sigma$ is equivariant as a section, $N \rightarrow T^{*} N$, and hence descends to a section, $\bar{\sigma}: N / G \rightarrow\left(T^{*} N\right) / G$, where $G$ acts on $T^{*} N$ via the lifted action.

Lemma 4.3. Suppose a Lie group $G$ acts on a line bundle $L \rightarrow X$ and $\sigma: X \rightarrow L$ is an invariant, non-vanishing section. Then $L$ is equivariantly trivial and hence $L / G \rightarrow X / G$ is a vector bundle.

Proof. The trivialization $X \times \mathbb{R} \rightarrow L$ defined by $(x, t) \mapsto t \cdot \sigma(x)$ is equivariant (where $G$ acts trivially on $\mathbb{R}$ ) since $\sigma$ is.

Proposition 4.4. Suppose a Lie group $G$ acts on a contact manifold $(M, \alpha)$, preserving $\alpha$, and let $\Phi_{\alpha}: M \rightarrow \mathfrak{g}^{*}$ be the associated moment map. Assume that $G$ acts locally freely and properly on the zero level set of $\Phi_{\alpha}$. Let $\xi=\operatorname{ker} \alpha$ be the contact distribution on $M$ and $\xi^{\circ}$ its annihilator in $T^{*} M$. Let $\xi^{\circ} / / G=\left(\xi_{\mid \Phi_{\alpha}^{-1}(0)}^{\circ}\right) / G$. Then there exists an embedding $\epsilon: \xi^{\circ} / / G \rightarrow T^{*}(M / / G)$ such that $\epsilon \circ \bar{\alpha}=\alpha_{0}$, where $\bar{\alpha}$ is the induced section and $\alpha_{0}$ is the reduced contact form on $M / / G$. 
Proof. Set $Z=\Phi_{\alpha}^{-1}(0)$. Then $Z$ is a submanifold of $M$ and the natural inclusion, $i: T Z \rightarrow T M_{\mid Z}$ gives rise to a projection, $i^{T}: T^{*} M_{\mid Z} \rightarrow T^{*} Z$. By Proposition 3.1 the Reeb vector field is tangent to $Z$. Split the tangent bundle of $M$ at $z$ as

$$
T_{z} M=\xi_{z} \oplus \mathbb{R} Y(z) .
$$

We obtain an induced splitting,

$$
T_{z} Z=\left(\xi_{z} \cap T_{z} Z\right) \oplus \mathbb{R} Y(z) .
$$

If $0 \neq \eta \in \xi_{z}^{\circ}$, then $\left\langle i^{T}(\eta), Y(z)\right\rangle \neq 0$. Therefore, $i^{T}$ embeds $\xi^{\circ}$ into $T^{*} Z$. Moreover, this embedding is equivariant.

Denote the symplectic moment map for the lifted $G$-action on $T^{*} Z$ by $\Psi$. Then

$$
\Psi^{-1}(0)=\left\{(z, \eta) \in T^{*} Z \mid\left\langle\eta, X_{Z}(z)\right\rangle=0 \text { for all } X \in \mathfrak{g}\right\} .
$$

For any $X \in \mathfrak{g}, z \in Z$, and $\eta \in \xi_{z}^{\circ}$, we have

$$
\left\langle i^{T}(\eta), X_{Z}(z)\right\rangle=\left\langle\eta, X_{M}(z)\right\rangle=0
$$

since $\Phi_{\alpha}(z)=0$ implies $X_{M}(z) \in \xi_{z}$ for all $X \in \mathfrak{g}$. Hence, $\iota^{T}$ embeds $\xi_{\mid Z}^{\circ}$ ) into $\Psi^{-1}(0)$. Let $\bar{\iota}^{T}: \xi^{\circ} / / G \rightarrow T^{*}(Z) / / G$ be the induced embedding. The co-tangent bundle reduction theorem of Abraham-Marsden and Kummer ( $\mathrm{AM}, \overline{\mathrm{Ku}}]$ ) asserts that there is a symplectomorphsim $\epsilon^{\prime}: T^{*}(Z) / / G \rightarrow T^{*}(M / / G)$. Set $\epsilon=\epsilon^{\prime} \circ \bar{\iota}^{T}$.

Recall that the reduced contact form on $M / / G$ is defined to be the unique 1form, $\alpha_{0}$, on $M / / G$ such that $\pi^{*}\left(\alpha_{0}\right)=\alpha_{\mid Z}$, where $\pi: Z \rightarrow M / / G$ is the orbit map. It follows, by definition, that $\pi^{*}(\epsilon \circ \bar{\alpha})=\alpha_{\mid Z}$, giving $\epsilon \circ \bar{\alpha}=\alpha_{0}$.

Since each stratum in the contact quotient at zero can be expressed as the reduction of a contact manifold under a free group action, our third theorem follows immediately.

Theorem 3. Suppose a Lie group $G$ acts on a contact manifold $(M, \alpha)$, preserving $\alpha$, and let $\Phi_{\alpha}: M \rightarrow \mathfrak{g}^{*}$ be the associated contact moment map. For each stabilizer subgroup, $H$, of $G$, let

$$
(M / / G)_{(H)}:=\left(\Phi_{\alpha}^{-1}(0) \cap M_{(H)}\right) / G
$$

denote the stratum associated to $H$. If $G$ acts properly on $\Phi_{\alpha}^{-1}(0)$, then there exists a line bundle $\xi^{\circ} / / G$ over $M / / G=\Phi_{\alpha}^{-1}(0) / G$ equipped with a section $\sigma$ such that for each such $H$, the restriction of $\xi^{\circ} / / G$ and $\sigma$ to $(M / / G)_{(H)}$ defines a co-oriented contact structure on the stratum.

Proof. Set $Z:=\Phi_{\alpha}^{-1}(0)$ and

$$
\xi^{\circ} / / G:=\left(\xi_{\mid Z}^{\circ}\right) / G
$$

By Lemma 4.3, $\xi^{\circ} / / G$ is a line bundle over $M / / G$. Let $N$ be the normalizer of $H$ in $G$ and $L=N / H$. Set

$$
M_{H}=\left\{m \in M \mid G_{m}=H\right\} .
$$

Then $M_{H}$ is a contact submanifold of $M$ and $L$ acts freely on $M_{H}$, preserving $\alpha_{\mid M_{H}}$. Denote the corresponding moment map by $\Psi_{\alpha}: M_{H} \rightarrow \mathfrak{l}^{*}$. Recall that we can identify $\Psi_{\alpha}^{-1}(0)$ with $\Phi_{\alpha}^{-1}(0) \cap M_{H}$ and that there is a diffeomorphism $\vartheta$ : $M_{H} / / L \rightarrow(M / / G)_{(H)}$. It follows that, without being too fussy about equivalences versus equalities,

$$
\left(\xi^{\circ} / / G\right)_{\mid(M / / G)_{(H)}}=\left(\xi_{\mid \Phi_{\alpha}^{-1}(0) \cap M_{(H)}}^{\circ}\right) / G=\left(\xi_{\mid \Psi_{\alpha}^{-1}(0)}^{\circ}\right) / L .
$$


Now apply the free case to obtain the result.

Proposition 3.12 and Theorems 2 and 3 immediately imply

Corollary 4.5. Suppose a Lie group $G$ acts on a contact manifold $(M, \alpha)$, preserving $\alpha$, and let $\Phi_{\alpha}: M \rightarrow \mathfrak{g}^{*}$ be the associated moment map. Choose $\mu \in \mathfrak{g}^{*}$ and suppose that

- $K_{\mu}$ acts properly on $\Phi_{\alpha}^{-1}\left(\mathbb{R}^{+} \mu\right)$;

- a convex slice exists for $\mu$ which is invariant under dilations by $\mathbb{R}^{+}$.

Let $K_{\mu}$ be the connected Lie subgroup of $G_{\mu}$ with Lie algebra $\mathfrak{k}_{\mu}=\operatorname{ker} \mu_{\mid \mathfrak{g}_{\mu}}$. For each stabilizer subgroup, $H$, of $G$, let

$$
\left(M_{\mu}\right)_{(H)}:=\left(\Phi_{\alpha}^{-1}\left(\mathbb{R}^{+} \mu\right) \cap M_{(H)}\right) / K_{\mu}
$$

denote the stratum associated to $H$. Then there exists a line bundle $\xi_{\mu}^{\circ}$ over

$$
M_{\mu}=\Phi_{\alpha}^{-1}\left(\mathbb{R}^{+} \mu\right) / K_{\mu}
$$

equipped with a section $\sigma$ such that for each such $H$, the restriction of $\xi_{\mu}^{\circ}$ and $\sigma$ to $\left(M_{\mu}\right)_{(H)}$ defines a co-oriented contact structure on the stratum.

\section{The Guillemin-Sternberg Quotient}

Let $G$ be a compact, connected Lie group and $\Theta$ a submanifold of $\mathfrak{g}^{*}$ which is invariant under the co-adjoint action of $G$ on $\mathfrak{g}^{*}$. Choose $\eta \in \Theta$ and let $\mathfrak{h}_{\eta}$ be the co-normal space of $\Theta$ at $\eta$. Then $\mathfrak{h}_{\eta}$ is a Lie ideal in $\mathfrak{g}_{\eta}$. Let $H_{\eta}$ be the unique connected Lie subgroup of $G_{\eta}$ with Lie algebra $\mathfrak{h}_{\eta}$. Call $\Theta$ proper if $H_{\eta}$ is closed in $G_{\eta}$ for all $\eta \in \Theta$.

Suppose that $G$ acts in a Hamiltonian fashion on a symplectic manifold $(N, \omega)$ and choose a corresponding equivariant moment map, $\Psi: N \rightarrow \mathfrak{g}^{*}$. Let $\Theta$ be a proper, invariant submanifold of $\mathfrak{g}^{*}$. If $\Psi$ is transverse to $\Theta$, then $Z:=\Psi^{-1}(\Theta)$ is a co-isotropic submanifold of $M$ and the leaf of the null foliation through $x \in Z$ is identified with the $H_{\Psi(x)}$ orbit through $x$.

Proposition 5.1 (Guillemin-Sternberg). Suppose a compact, connected Lie group $G$ acts in a Hamiltonian fashion on a symplectic manifold $(N, \omega)$ and let $\Psi: N \rightarrow \mathfrak{g}^{*}$ be a corresponding equivariant moment map. Let $\Theta \subset \mathfrak{g}^{*}$ be an invariant, proper submanifold of $\mathfrak{g}^{*}$ and set $Z:=\Psi^{-1}(\Theta)$. If $\Psi$ is transverse to $\Theta$, then the leaf space,

$$
N_{\Theta}:=Z / \sim \quad \text { where } p \sim q \text { if and only if } p \text { and } q \text { are on the same leaf }
$$

is a symplectic orbifold.

The reader is refered to [GS1] for a proof of this Proposition.

Example 5.2. If $\Theta=0 \in \mathfrak{g}^{*}$, then $N_{\Theta}$ is the Marsden-Weinstein-Meyer reduced space. If $\Theta=\mathcal{O}$, a co-adjoint orbit, then $N_{\Theta}$ is the Kahzdan-Kostant-Sternberg reduced space.

Definition 5.3. A symplectic manifold $(N, \omega)$ is called a symplectic cone if there is a free, proper $\mathbb{R}$-action $\tau: N \times \mathbb{R} \rightarrow N$ such that $\tau_{t}^{*} \omega=e^{t} \omega$.

Example 5.4. Let $(M, \alpha)$ be a compact, co-oriented contact manifold and $(M \times$ $\left.\mathbb{R}, d\left(e^{t} \alpha\right)\right)$ the symplectization of $(M, \alpha)$. Then the action of $\mathbb{R}$ on $M \times \mathbb{R}$ given by $s \cdot(m, t)=(m, t+s)$ makes $\left(M \times \mathbb{R}, d\left(e^{t} \alpha\right)\right)$ into a symplectic cone. 
In fact, one can show that all symplectic cones are of the form given in the above example.

Proposition 5.5. Let $(N, \omega)$ be a symplectic cone. Then $N \simeq M \times \mathbb{R}$ where $M=$ $N / \mathbb{R}$ is a co-oriented contact manifold.

The reader is referred to, for example, L2] for a proof of the above proposition and more details on symplectic cones.

Definition 5.6. Call $\mu \in \mathfrak{g}^{*}$ integral if there is a group homomorphism $\chi_{\mu}$ : $G_{\mu} \rightarrow S^{1}$ such that $d \chi_{\mu}=\mu_{\mid \mathfrak{g}_{\mu}}$. Call a co-adjoint orbit $\mathcal{O}$ an integral orbit if it is an orbit through an integral element of $\mathfrak{g}^{*}$.

Remark 5.7. Note that if $\mu$ is integral, then $H_{\mu}=$ ker $\chi_{\mu}=K_{\mu}$, the kernel group of $\mu$ defined earlier. Integral orbits are automatically proper. The cone on an integral orbit $\mathbb{R}^{+} \mathcal{O}$ is a proper submanifold of $\mathfrak{g}^{*}$.

Let $(M, \alpha)$ be a co-oriented contact manifold equipped with an action of a compact, connected Lie group $G$ which preserves $\alpha$ and let $\Phi_{\alpha}: M \rightarrow \mathfrak{g}^{*}$ be the associated contact moment map. Extend the $G$-action to the symplectization $\left(M \times \mathbb{R}, d\left(e^{t} \alpha\right)\right)$ by $g \cdot(m, t)=(g \cdot m, t)$. A corresponding equivariant symplectic moment map

$$
\Psi: M \times \mathbb{R} \rightarrow \mathfrak{g}^{*}
$$

is given by $\Psi(m, t)=e^{t} \Phi_{\alpha}(m)$. Let $\mathcal{O} \subset \mathfrak{g}^{*}$ be an integral orbit and assume that $\Psi$ is transveral to $\mathbb{R}^{+} \mathcal{O}$. By Proposition 5.1, $(M \times \mathbb{R})_{\mathbb{R}^{+} \mathcal{O}}$ is a symplectic orbifold. The $\mathbb{R}$-action on $M \times \mathbb{R}$ described in Example 5.4 descends to $(M \times \mathbb{R})_{\mathbb{R}+\mathcal{O}}$. By Proposition 5.5, the orbit space under the $\mathbb{R}$-action on $(M \times \mathbb{R})_{\mathbb{R}+\mathcal{O}}$ is thus a contact manifold. I call it the Guillemin-Sternberg reduction of $M$ by $\mathcal{O}$. It is denoted by $M_{\mathcal{O}}^{G S}$.

The Guillemin-Sternberg reduction should be thought of as a larger form of contact reduction in the following sense. Since $\mathbb{R}^{+} \mathcal{O}$ is $\mathbb{R}^{+}$-invariant, $\Psi^{-1}\left(\mathbb{R}^{+} \mathcal{O}\right)=$ $\Phi_{\alpha}^{-1}\left(\mathbb{R}^{+} \mathcal{O}\right) \times \mathbb{R}$. For any $\mu \in \mathcal{O}$ the contact quotient $M_{\mu}$ is a contact suborbifold of $M_{\mathcal{O}}^{G S}$. More, in fact, is true. Since the leaf of the null foliation through $(p, t)$ is given by the $K_{\Phi_{\alpha}(p)}$ orbit through $(p, t)$, the restriction of $\Psi$ to a leaf in $\Psi^{-1}\left(\mathbb{R}^{+} \mathcal{O}\right)$ is constant. Therefore, $\Psi$ descends to

$$
\tilde{\Psi}:(M \times \mathbb{R})_{\mathbb{R}^{+\mathcal{O}}} \rightarrow \mathbb{R}^{+} \mathcal{O} .
$$

For any $t \in \mathbb{R}$, let $\epsilon_{t}^{\mu}: \Phi_{\alpha}^{-1}\left(\mathbb{R}^{+} \mu\right) \rightarrow \mathbb{R}$ be the map $m \mapsto t-t_{m}$, where $\Phi_{\alpha}(m)=$ $e^{t_{m}} \mu$. Denote the graph of $\epsilon_{t}^{\mu}$ by $\Gamma_{t}^{\mu}$. It follows that $\Gamma_{t}^{\mu}=\Psi^{-1}\left(e^{t} \mu\right)$ and, since $K_{e^{s} \mu}=K_{e^{t} \mu}$, that

$$
\tilde{\Psi}^{-1}\left(e^{t} \mu\right)=\Gamma_{t}^{\mu} / K_{\mu} \simeq M_{\mu} .
$$

In summary, we have shown

Proposition 5.8. Let $(M, \alpha)$ be a compact, connected, co-oriented contact manifold equipped with an action of a compact, connected Lie group $G$ which preserves $\alpha$ and let $\Phi_{\alpha}: M \rightarrow \mathfrak{g}^{*}$ be the associated moment map. Choose an integral $\mu \in \mathfrak{g}^{*}$ and let $\mathcal{O}$ be the co-adjoint orbit through $\mu$. Then $M_{\mathcal{O}}^{G S}$ fibers over $\mathcal{O}$ with typical fiber $M_{\mu}$. 


\section{Appendix A. Albert's quotient}

Independent of Guillemin-Sternberg, C. Albert discovered an elegant method for contact reduction. However, his method depends upon the choice of contact form used to represent the given contact structure. Let $(M, \alpha)$ be a co-oriented contact manifold on which a compact group $G$ acts by contact transformations. Let $\Phi_{\alpha}: M \rightarrow \mathfrak{g}^{*}$ be the associated moment map. Suppose $\mu$ is a regular value of $\Phi_{\alpha}$, so that $Z:=\Phi_{\alpha}^{-1}(\mu)$ is a submanifold of $M$. Consider the map, $\tilde{\tau}: \mathfrak{g}_{\mu} \rightarrow \chi(Z)$ defined by $A \mapsto A_{Z}-\langle\mu, A\rangle Y$, where $Y$ is the Reeb vector field and $\chi(Z)$ is the algebra of vector fields on $Z$. It follows that $\tilde{\tau}$ is a map of Lie algebras. By a theorem of Palais there is a unique map, $\tau: \tilde{G}_{\mu} \rightarrow \operatorname{Diff}(Z)$, where $\tilde{G}_{\mu}$ is the universal covering group of $G_{\mu}$, whose differential is $\tilde{\tau}$. Set $H=\tilde{G}_{\mu} / \operatorname{ker} \tau$. Then $H$ acts locally freely and effectively on $Z$. The Albert reduction of $M$ by $G$ at $\mu$ is defined to be

$$
M_{\mu}^{A}:=Z / H
$$

If the $H$ action is proper, this is an orbifold and, by definition, $\alpha$ descends to $\alpha_{\mu}$ on $M_{\mu}^{A}$. A standard argument shows that $\alpha_{\mu}$ is a contact form. However $f \alpha$, where $f$ is a positive invariant function, is an invariant contact form with associated moment map $\Phi_{f \alpha}=f \Phi_{\alpha}$. This indicates that the Albert quotient will be dependent on the choice of contact form. The following example illustrates this dependence.

Example A.1. Let

$$
\begin{aligned}
& \mathcal{E}_{1}=\left\{\left.\left(z_{1}, z_{2}, z_{3}\right) \in \mathbb{C}^{3}|| z_{1}\right|^{2}+2\left|z_{2}\right|^{2}+2\left|z_{3}\right|^{2}=1\right\}, \\
& \mathcal{E}_{2}=\left\{\left.\left(z_{1}, z_{2}, z_{3}\right) \in \mathbb{C}^{3}\left|\frac{1}{2}\right| z_{1}\right|^{2}+\left|z_{2}\right|^{2}+\frac{1}{3}\left|z_{3}\right|^{2}=1\right\} .
\end{aligned}
$$

Let $S^{1}$ act on $\mathcal{E}_{i}, i=1,2$, by the restriction of the action on $\mathbb{C}^{3}$ given by the weights $(1,-1,-1)$. Since both $\mathcal{E}_{i}$ are star shaped about the origin in $\mathbb{C}^{3}$, they are isomorphic contact manifolds. The difference in shape amounts to a choice of different contact forms in the same conformal class. Let $\Phi_{i}$ denote the contact moment map for the $S^{1}$ action on $\mathcal{E}_{i}$. A simple calculation yields

$$
\Phi_{1}\left(z_{1}, z_{2}, z_{3}\right)=\Phi_{2}\left(z_{1}, z_{2}, z_{3}\right)=\left|z_{1}\right|^{2}-\left|z_{2}\right|^{2}-\left|z_{3}\right|^{2} .
$$

It is not hard to see that

$$
\Phi_{1}^{-1}(1)=\left\{\left.\left(z_{1}, 0,0\right) \in \mathbb{C}^{3}|| z_{1}\right|^{2}=1\right\}
$$

and that the Albert action is trivial. Hence the reduction of $\mathcal{E}_{1}$ is the circle, $S^{1}$.

A more tedious calculation gives

$$
\begin{gathered}
\Phi_{2}^{-1}(1)=\left\{\left.\left(z_{1}, z_{2}, z_{3}\right) \in \mathbb{C}^{3}|| z_{1}\right|^{2}=\frac{2}{3}\left(2+\frac{2\left|z_{3}\right|^{2}}{3}\right),\left|z_{2}\right|^{2}=-\frac{2}{3}\left(-\frac{1}{2}+\frac{5\left|z_{3}\right|^{2}}{6}\right),\right. \\
\left.0 \leq\left|z_{3}\right|^{2} \leq \frac{3}{5}\right\} .
\end{gathered}
$$

The symmetry group in the Albert quotient is the circle, acting with weights $\left(\frac{1}{2},-2,-\frac{4}{3}\right)$. Note that $\Phi_{2}^{-1}(1)$ contains a 3 -torus, namely

$$
\left\{\left(z_{1}, z_{2}, z_{3}\right) \in \mathbb{C}^{3}|| z_{1}^{2}=\frac{14}{9},\left|z_{2}\right|^{2}=\frac{1}{18},\left|z_{3}\right|^{2}=\frac{1}{2}\right\} .
$$

Hence, the Albert reduction of $\mathcal{E}_{2}$ is at least 3 dimensional, illustrating the dependence of the Albert quotient on the choice of the contact form. 


\section{REFERENCES}

[AM] R. Abraham and J. E. Marsden, Foundations of Mechanics, 2nd Ed., Addison-Wesley, 1978. MR 81e:58025

[Al] C. Albert, Le théorème de réduction de Marsden-Weinstein en géométrie cosymplectique et de contact, J. Geom. Phys. 6 (1989), no. 4, 627-649. MR 91k:58033

[BL] L. Bates and E. Lerman, Proper group actions and symplectic stratified spaces, Pac. J. of Math. (2) 181 (1997), 201-229. MR 98i:58085

[Ge] H. Geiges, Constructions of contact manifolds, Math. Proc. Cambridge Philos. Soc. 121 (1997), no. 3, 455-464. MR 98f:53027

[GS1] V. Guillemin and S. Sternberg, Homogeneous quantization and multiplicities of group representations, J. Funct. Anal. 47 (1982), no. 3, 344-380. MR 84d:58034

[GS2] V. Guillemin and S. Sternberg, Symplectic Techniques in Physics, Cambridge University Press, Cambridge, 1984. MR 86f:58054

$[\mathrm{Ku}] \quad$ M. Kummer, On the construction of the reduced phase spaces of a Hamiltonian system with symmetry, Indiana Univ. Math. J. 30 (1981), no. 2, 281-291. MR 82e:58041

[L1] E. Lerman, Contact Cuts, Israel J. Math 124 (2001), 77-92. See http://xxx.lanl.gov/abs/math.SG/0002041.

[L2] E. Lerman, Geodesic flows and contact toric manifolds, CRM Summer School in the Symplectic Geometry of Integrable Hamiltonian System, Notes. See http://front.math.ucdavis.edu/math.SG/0201230

[LMTW] E. Lerman, E. Meinrenken, S. Tolman, and C. Woodward, Non-abelian convexity by symplectic cuts, Topology 37 (1998), no. 2, 245-259. MR 99a:58069

[LW] E. Lerman and C. Willett, The topological structure of contact and symplectic quotients, Internat. Math. Res. Notices (2001), no. 1, 33-52. MR 2001j:53112

[Lo] F. Loose, Reduction in contact geometry, J. Lie Theory 11 (2001), no. 1, 9-22. CMP 2001:12

[P] R. Palais, On the existence of slices for actions of non-compact Lie groups, Ann. Math. 73 (1961), no. 2, 295-322. MR 23;A3802

Department of Mathematics, University of Illinois, Urbana, Illinois 61801

E-mail address: cwillett@math.uiuc.edu 